\title{
Tactile Texture Display with Vibrotactile and Electrostatic Friction Stimuli Mixed at Appropriate Ratio Presents Better Roughness Textures
}

\author{
KEN ITO, SHOGO OKAMOTO, and YOJI YAMADA, Nagoya University, Japan \\ HIROYUKI KAJIMOTO, University of Electro-Communication, Japan
}

\begin{abstract}
Vibrotactile and friction texture displays are good options for artificially presenting the roughness and frictional properties of textures, respectively. These two types of displays are compatible with touch panels and exhibit complementary characteristics. We combine vibrotactile and electrostatic friction texture displays to improve the quality of virtual textures, considering that actual textured surfaces are composed of both properties. We investigate their composition ratios when displaying roughness textures. Grating roughness scales with one of the six surface wavelengths are generated under 11 display conditions, and in 9 of which, vibrotactile and friction stimuli are combined with different composition ratios. A forced-choice experiment regarding subjective realism indicates that a vibrotactile stimulus with a slight variable-friction stimulus is effective for presenting quality textures for surface wavelengths greater than or equal to $1.0 \mathrm{~mm}$.
\end{abstract}

CCS Concepts: • Human-centered computing $\rightarrow$ Laboratory experiments; Empirical studies in HCI; • Hardware $\rightarrow$ Haptic devices;

Additional Key Words and Phrases: Tactile texture display, vibrotactile display, electrostatic friction display, surface roughness

ACM Reference format:

Ken Ito, Shogo Okamoto, Yoji Yamada, and Hiroyuki Kajimoto. 2019. Tactile Texture Display with Vibrotactile and Electrostatic Friction Stimuli Mixed at Appropriate Ratio Presents Better Roughness Textures. ACM Trans. Appl. Percept. 16, 4, Article 20 (September 2019), 15 pages.

https://doi.org/10.1145/3340961

\section{INTRODUCTION}

Tactile displays have received a considerable amount of attention with the widespread use of touch panel devices. This study deals with the presentation of virtual textures using tactile displays installed on touch panels. Tactile displays suitable for commercial touch panels are divided into vibrotactile-type (e.g., References $[2,11,17,38,57]$ ) and friction-type displays (e.g., References [4, 15, 35, 49, 58]). The former type of display mechanically deforms the finger pad by driving the touch panel, and the latter type of display controls the panel friction. Vibrotactile stimuli

The work was supported by MEXT Kakenhi under Grants No. 15H05923 and No. 17 H04697.

Authors' addresses: K. Ito, S. Okamoto (corresponding author), and Y. Yamada, Nagoya University, Furo-cho, Chikusa-ku, Nagoya, Japan, 4648603; emails: itou.ken@a.mbox.nagoya-u.ac.jp, okamoto-shogo@nagoya-u.jp, yamada-yoji@mech.nagoya-u.ac.jp; H. Kajimoto, University of Electro-Communication, 1-5-1 Nunogaoka, Chofu, Tokyo, Japan; email: kajimoto@hc.uec.ac.jp.

Permission to make digital or hard copies of all or part of this work for personal or classroom use is granted without fee provided that copies are not made or distributed for profit or commercial advantage and that copies bear this notice and the full citation on the first page. Copyrights for components of this work owned by others than ACM must be honored. Abstracting with credit is permitted. To copy otherwise, or republish, to post on servers or to redistribute to lists, requires prior specific permission and/or a fee. Request permissions from permissions@acm.org.

(c) 2019 Association for Computing Machinery.

1544-3558/2019/09-ART20 \$15.00

https://doi.org/10.1145/3340961

ACM Transactions on Applied Perception, Vol. 16, No. 4, Article 20. Publication date: September 2019. 
are suitable for controlling the sense of a surface roughness feature. Variable friction displays aim to represent the friction distribution on a surface and frictional vibration. Both types of displays can evoke a sense of textured surfaces such as grating scales [2, 38, 55, 58] and roughened surfaces [32]. A question is then naturally raised. Does the integration of vibrotactile and variable friction stimuli improve virtual textures in terms of realism? For the perception of fine roughness textures, skin vibration is the important cue $[6,20,56]$, and both types of texture displays can induce skin vibration. Hence, the two types of stimuli may present textures with similar realism, and the combined stimuli do not substantially improve realism, because both types of stimuli can generate skin vibration. In contrast, it could be argued that the combination of multiple modalities is effective for resembling realistic surface contact states.

The tactile perception of surfaces is expressed in a multidimensional space [5, 7, 28, 37]. Haptic displays that control multiple modalities have been developed thus far to artificially present such perception [9, 11, 25, 30, $41,60]$. In this article, texture is referred to as surfaces featuring roughness and friction. Roughness and friction perception are considered to be perceptually independent [3, 16, 19, 46, 61]; however, friction physically depends on the surface roughness. Both macroscopic and microscopic surface roughnesses influence the friction forces when a finger scans the surface [13, 47, 54]. For example, surface asperities influence frictional forces applied to the finger pad when the finger slides over a surface [12, 14, 24]. Hence, the senses of roughness and friction always arise when exploring textured surfaces. The roles of roughness and friction for texture perception and material discrimination have not been fully understood partly because of the difficulties associated with independent control or the separation of surface roughness and friction. In psychophysical experiments involving multiple modalities, highly controlled stimuli are generally required; however, decoupling the surface roughness and friction is demanding. Although lubrication is a method for weakening the adhesion between the finger pad and the textured surface $[1,52]$, deformation friction and ploughing are rarely affected by lubrication. Moreover, the effect of the viscosity of the lubricant must be considered when using lubrication.

The objective of the present study is to investigate whether a texture display combining vibrotactile and variable-friction stimuli presents better texture stimuli. Thus far, no studies except for our previous ones [22, 23] have attempted to combine the two types of stimuli for developing quality tactile textures; however, one study reported a tactile display that could deliver both types of stimuli $[41,43]$ using vibrotactile and friction stimuli separately for presenting fine textures and macroscopic surface profiles, respectively. The friction distribution on a plane leads to the perception of macroscopic surface profiles such as bumps and holes [26, 39, 42]. The combination of frictional and vibrotactile stimuli embodies a variety of functional haptic feedbacks and improves user experiences [31]. In addition, the perceptual interference between two types of stimuli was reported [41, 44] where intense vibration masked the perception of electrostatic frictional vibration of detection levels. Another study synchronized electrostatic attraction and lateral vibration to generate a lateral force on a plane [34]. These studies indicated that a joint technique of passive frictional stimuli and active vibrotactile stimuli broadens the possibilities of tactile displays.

Our previous experiments [22, 23] indicated that the combination of two types of tactile stimulations is effective for improving the realism of virtual roughness textures. In the experiments, participants perceived virtual grating scales under three conditions: textures presented by vibrotactile stimuli, electrostatic friction, and the conjunction of vibrotactile and friction stimuli. While comparing the virtual and actual grating scales, the participants selected one of the three conditions in an alternative-forced-choice manner. The condition in which the two were combined was judged to be the most realistic by the majority of participants. In this study, we investigate the composition ratio of the two types of tactile stimulations for achieving better realism when the spatial wavelengths of virtual grating scales vary between 0.5 and $3.0 \mathrm{~mm}$. In other words, we compared 11 conditions, 9 of which are conjunction conditions with different vibrotactile-electrostatic composition rates whereas our previous studies $[22,23]$ tested only one conjunction condition. The acquisition of a better composition ratio for each roughness scale would contribute to the design of the hardware and rendering algorithms of tactile texture displays. 


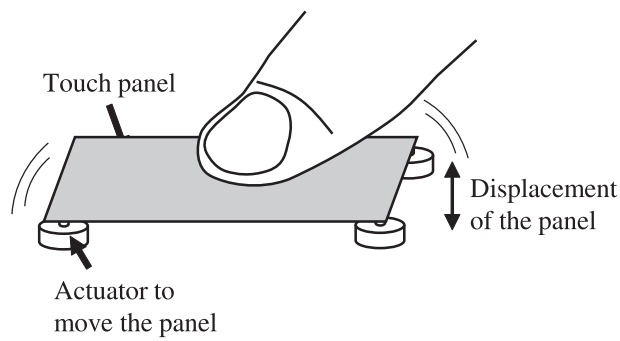

(a) Vibrotactile display

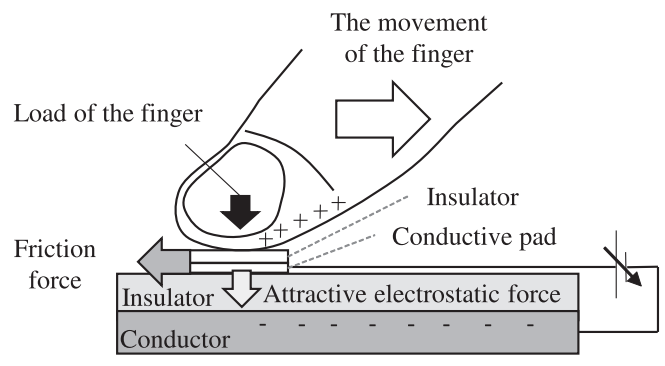

(b) Electrostatic friction display

Fig. 1. Principles of vibrotactile and electrostatic friction displays (modified from Reference [22]).

\section{VIBROTACTILE AND ELECTROSTATIC FRICTION DISPLAYS}

Both vibrotactile and electrostatic friction displays can be mounted on a touch panel. The characteristics for each type of display are summarized below. Previous studies on tactile displays and products mostly use either only vibrotactile-type or only friction-type displays. As aforementioned, a texture is composed of the surface roughness and friction features. Hence, the combination of roughness-oriented vibrotactile stimuli and friction stimuli is a reasonable way to represent texture stimuli.

\subsection{Vibrotactile Display}

Figure 1(a) shows the principles of a vibrotactile display. A touch panel is mechanically driven or vibrated by a set of high-fidelity actuators such as voice coil motors and piezoelectric devices. A finger pad on the touch panel is then deformed to induce tactile sensations. Tactile stimuli are typically presented along the normal to the finger pad. This display is a good option for depicting surface-roughness textures [2, 11, 29, 38, 57]; nonetheless, natural friction accrues between the flat panel and the finger pad when the finger slides on the panel. The touch panel itself actively produces mechanical stimulation; hence, for presenting tactile stimuli, this display does not necessarily need active finger movement. However, tactile stimuli synchronized with the movement are considered to be a more natural form of tactile feedback.

\subsection{Electrostatic Friction Display}

Figure 1(b) shows the principles of an electrostatic friction display. This display controls the friction between a finger pad and a touch panel using the attractive force caused by the electric charge between the touch panel and the finger pad. The friction changes by controlling the voltage applied between the finger pad and the panel, and thus the attractive force; then, the variation in the surface friction is simulated on the panel. This display is generally a good option for depicting a texture dominated by friction $[4,35]$ and a macrosurface pattern [27, 39]. 


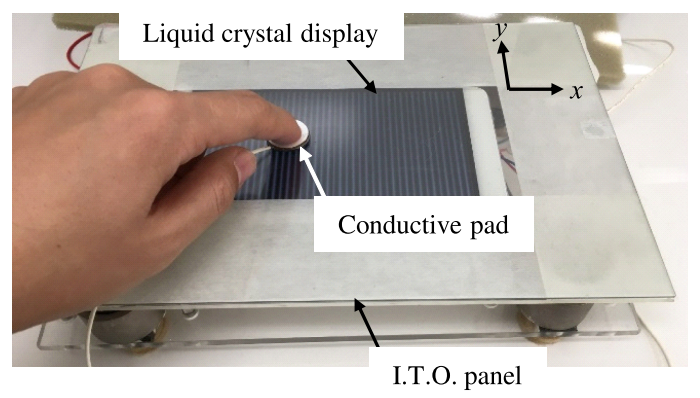

(a) Display image. The texture image is not shown by the liquid crystal display during the experiment.

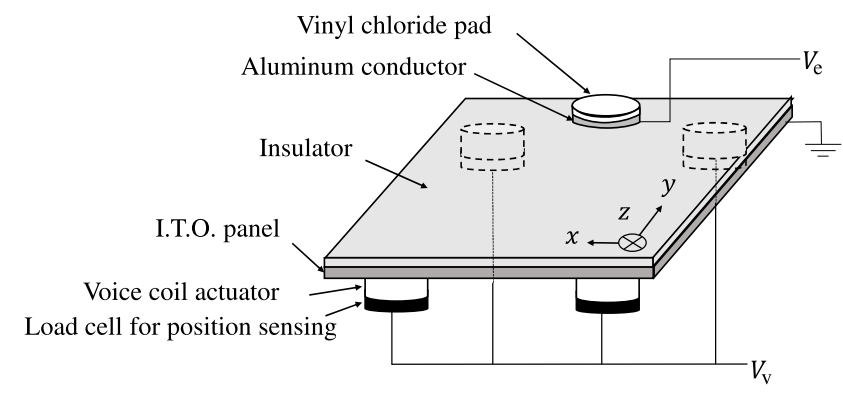

(b) Schematic of the structure (modified from [22])

Fig. 2. Overall structure of the tactile display for vibrotactile and electrostatic friction stimuli.

As shown in Figure 1(b), in our experimental setup, the voltage was applied to the conductive pad to control the contact condition between the finger pad and the panel for the purposes of psychophysical experiments. Such a conductive pad also allows us to produce large attractive forces and resultant friction forces with relatively small applied voltages, whereas the sense of frictional variation is slightly different between conditions with and without the conductive pad.

\section{APPARATUS: TACTILE TEXTURE DISPLAY USING VIBROTACTILE AND ELECTROSTATIC FRICTION STIMULI}

We used a tactile display that was developed in our previous studies [22,23] and is shown in Figure 2. This display simultaneously presents vibrotactile and electrostatic friction stimuli. We used actuators and amplifiers that could deliver output stimuli far above the threshold levels for perception, because this was for a laboratory prototype. Although the apparatus can present a visual image beneath the transparent panel, no textural images were shown.

Vibrotactile stimuli were produced by four voice coil actuators (X-1741, Neomax Engineering Co. Ltd., Japan, maximum output force: $6 \mathrm{~N}$ ) located at the four corners of the top panel. The four actuators were synchronously driven by a current amplifier (ADS 50/5, Maxon Motor AG, Switzerland) and mechanical stimuli were produced along the normal to the top panel. The mass of the top panel was $283 \mathrm{~g}$. The natural frequency of the normal motion was $25 \mathrm{~Hz}$, around which mechanical resonance occurs; however, this range was rarely used in the experiments mentioned later. 


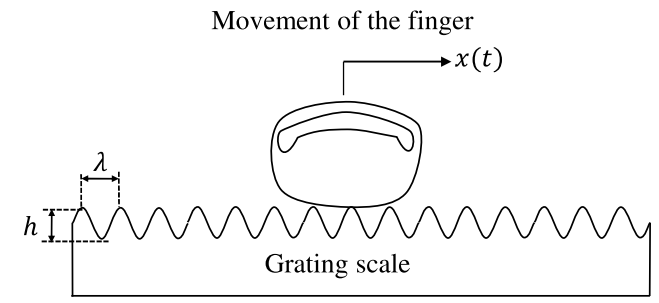

(a) Sinusoidal grating scale and the definitions of $\lambda$ and $h . x(t)$ is the finger position at $t$.

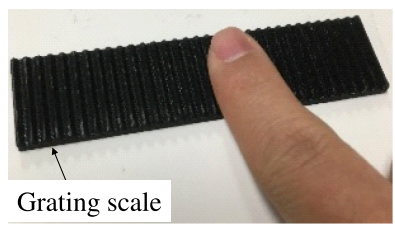

(b) Image of an ABS grating scale.

Fig. 3. Grating scale (roughness scale) with a sinusoidal surface.

An electrostatic friction force was generated between the indium tin oxide (ITO) panel layer and the conductive pad by applying a voltage $\left(V_{\mathrm{e}}\right)$ between them (Figure 2(b)). An insulator (Kimotect PA8X, KIMOTO Co. Ltd., Japan, $8 \mu \mathrm{m}$ ) was fixed on the ITO plate. $V_{\mathrm{e}}$ was driven by a high-voltage amplifier (HJOPS-1B20, Matsusada Precision Inc., Japan, maximum output: $\pm 1 \mathrm{kV}$, response: $75 \mathrm{kHz}$ ). A circular aluminum pad of $2 \mathrm{~mm}$ in thickness and $16 \mathrm{~mm}$ in diameter was introduced to qualify the large frictional forces and regulate the contact condition on the panel with no respect to individual finger size and its moisture condition. The pad directly transfers vibrotactile stimuli from the panel to the finger pad because of its rigidity and does not change the experience of vibrotactile stimuli when both vibrotactile and electrostatic stimuli exist. The surface friction between the panel and the conductive pad was small, and the coefficient of friction was smaller than 0.1 when the normal load was $1 \mathrm{~N}$.

The position and velocity of a finger were detected by four load cells (FS2050-0000-1500-G, TE Connectivity, Switzerland); each of these was affixed beneath each voice coil motor. The signal from each cell passed through an analog low-pass-filter circuit to filter the effects of high-frequency noise and the mechanical vibration of the system. The center of the force on the panel was calculated using the four load cells and considered to be the finger position on the panel. The resultant positions involved static errors of which standard deviation was $1.0 \mathrm{~mm}$ after low-pass filtering signal processing.

The two amplifiers and four load cells were connected to a data acquisition board (TNS-6812, Interface Corporation, Japan) controlled by a personal computer at $2 \mathrm{kHz}$. Our display presented tactile stimuli based on the finger motion, and with no finger movement, no tactile stimuli were provided.

\section{EXPERIMENT}

\subsection{Actual Texture: Sinusoidal Grating Scale}

We adopted a sinusoidal grating scale with periodic surface patterns (Figure 3) as reference stimuli during experiments. Trapezoidal or rectangular scales have local edges and are not effectively presented by our setup with the flat touch panel. The scale was made of ABS resin defined by a spatial wavelength $\lambda$ and had a wave depth of $h$ (Figure 3(a)). The effects of the combined texture stimuli depend on $\lambda$ [22, 23]; hence, we considered six levels of spatial wavelength $(\lambda=0.5,1.0,1.5,2.0,2.5$, and $3.0 \mathrm{~mm}) . h$ was $1.0 \mathrm{~mm}$ when $\lambda=0.5-2.0$ and $2.0 \mathrm{~mm}$ when 
$\lambda=2.5$ or $3.0 \mathrm{~mm}$. We determined the depths of the wave such that the finger pad did not reach the bottom of the scale to avoid the effects of shallow inter-element spaces [50,52], which our and extant texture rendering algorithms do not address. The surfaces of the grating scales were finely finished. As mentioned later, participants could freely touch the actual texture as a reference during experiments.

\subsection{Virtual Roughness Texture}

Normal (vibrotactile) and friction forces were output by the tactile texture display to present the virtual grating scales. We adopted rendering methods for each type of force from previous studies [2, 55, 60]. The two types of stimuli were synchronized in a spatiotemporal manner, because they referred to the same temporal and spatial bases (i.e., $t$ and $x$ in the equations that follow).

4.2.1 Vibrotactile Stimulus. We determined the driving force by voice coil motors as follows to simulate the displacement of a sinusoidal grating scale or the skin vibration when the finger pad slides on it:

$$
F_{\mathrm{v}}(t)=A \sin 2 \pi \frac{x(t)}{\lambda}
$$

where $x(t)$ and $A$ denote the position of the finger pad on the panel and the maximum output force, respectively. This formula determines the vibrotactile stimulus corresponding to finger movement.

4.2.2 Electrostatic Friction Stimulus. When a finger slides on a bump on the surface, the friction force is a function of the gradient of the surface displacement [14, 42]. In other words, the phase of the frictional force and surface displacement on a sinusoidal grating scale is $\pi / 2 \mathrm{rad}$. Hence, the applied voltage for the electrostatic friction stimulation is determined as follows:

$$
V_{\mathrm{e}}(t)=B \cos 2 \pi \frac{x(t)}{2 \lambda}
$$

where $B$ is the maximum voltage. The friction force between the finger and the panel is determined as follows by the law of electrostatic force and Coulomb's law of friction:

$$
\begin{aligned}
F_{\mathrm{e}}(t) & =\mu\left\{W+k V_{\mathrm{e}}^{2}(t)\right\} \\
& =\mu\left\{W+\frac{k B^{2}}{2}\left(1+\cos 2 \pi \frac{x(t)}{\lambda}\right)\right\},
\end{aligned}
$$

where $\mu, W$, and $k$ denote the coefficient of friction, the normal load of the finger, and a constant of the electrostatic friction force, respectively. $k$ mainly depends on the contact area of the conductive pad and the relative dielectric constant of the insulator and its thickness. The first term in the braces is related to the finger load, while the second term is related to the electrostatic attractive force. We confirmed that the friction force followed this quadratic formula by using the DC voltages by using the method described in Section 4.2.3. ${ }^{1}$

4.2.3 Stimuli with Different Composition Ratios of Vibrotactile and Friction Stimuli. We focused on the composition ratio of the vibrotactile and electrostatic friction stimuli in this experiment. In other words, we investigated what combination of two stimuli will present a highly realistic texture. For this purpose, two wide-ranging and continuous variables, which are the magnitudes of the vibrotactile and friction forces, have to be investigated. However, classical psychophysical methods are not available in a straightforward manner for such purposes. For instance, the two-alternative forced-choice task that is one of the most mathematically grounded approaches produces a large number of combinations to be tested when it is applied to a two-variable problem. Further, the

\footnotetext{
${ }^{1}$ It is likely that the quadratic relationship between the friction force and applied voltage also holds even for AC voltages. We could not install the precise force sensing element on the apparatus and measure the dynamic frictional force; however, the peak acceleration values measured on the conductive pad quadratically changed as a function of $B$.
} 


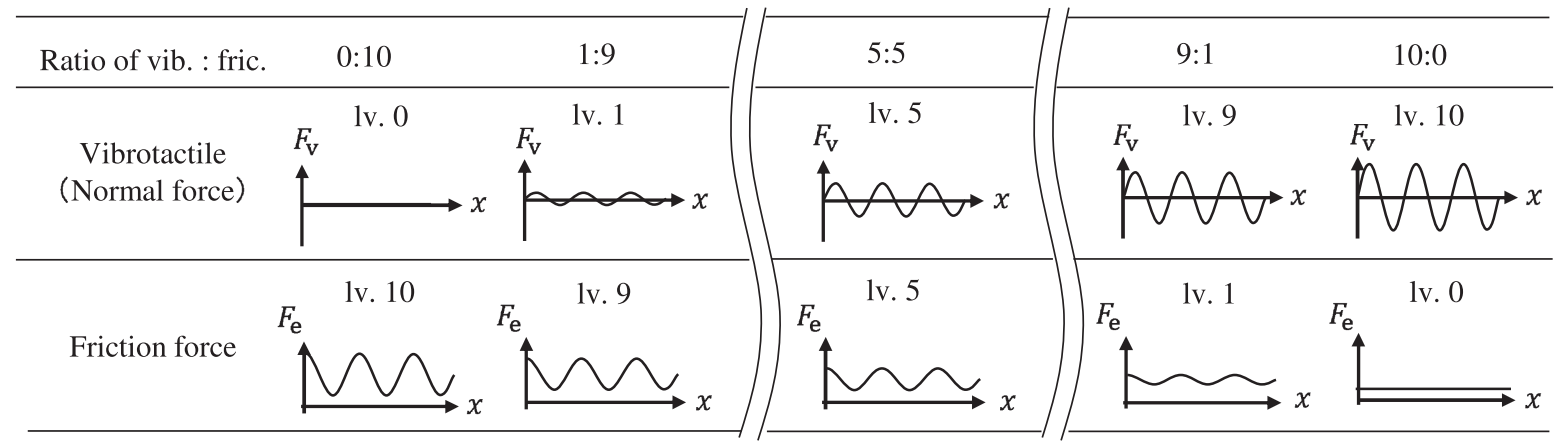

Fig. 4. Combination ratios of vibrotactile and friction stimuli. At level 10, the strength is tuned such that the stimulus is felt realistic when either of the vibrotactile or electrostatic friction stimuli is presented. The stimulus is just perceivable at level 1 but not present at level 0 . The strength is linearly determined in terms of levels 2-9.

method of adjustment is usually to manipulate one parameter. Hence, we adopted an $m$-alternative forced-choice task $[10,33,36,53]$ that is an expansion of the two-alternative forced-choice task. We prepared for a set of $m$ possible composition ratios, and participants selected the one that felt most realistic. This may not be a sophisticated method compared with classical ones especially in terms of statistical tests; however, our objective would be accomplished by this approach.

First, we tuned gains $A$ and $B$ for each spatial wavelength of the grating scale such that each stimulus was most realistic when considering only vibrotactile or electrostatic friction. These values of $A$ and $B$ were regarded as level 10 for each type of stimulus. The smallest stimulus felt through our tactile texture display was level 1. These settings were attained through consensus by five experienced people, including those conducting the experiments. Each of them determined the values for levels 1 and 10 by the method of adjustment. Their average values were then used as the final values. Levels 2-9 were determined by dividing the values of the output force between levels 1 and 10. In this way, 11 levels of stimuli were prepared for both vibrotactile and electrostatic friction stimuli. Among the 11 levels, level 0 denoted no stimuli. The stimuli sets for all 11 combinations of vibrotactile and electrostatic friction stimuli were prepared as follows for each spatial wavelength of the grating scale: $0: 10,1: 9,2: 8,3: 7,4: 6,5: 5,6: 4,7: 3,8: 2,9: 1$, and 10:0. Figure 4 shows an image of some of these combinations. The perceived magnitudes experienced from these 11 stimuli were comparable. In contrast, combinations such as 10:10 and 9:9 induced subjective magnitudes greater than the above 11 stimuli set. Similarly, combinations such as 1:1 and 2:2 were perceived as weak. Hence, stimuli that felt stronger or weaker than the best tuned stimuli (i.e., 10:0 and 0:10) were excluded from the test stimuli.

In our previous reports [22, 23], the number of stimulus conditions was 3 . In the present study, we prepared 11 conditions to investigate the effects of the composition ratios in detail, but other numbers of levels can also be used. We linearly split the stimuli on the basis of physical quantity, i.e., force. Another option is separation based on perceptual indices, such as a discrimination limen. In the latter case, the stimulus quantities are nonlinearly divided, while we used linearly separated stimuli.

Table 1 lists the maximum values of the vibrotactile force $F_{\mathrm{v}}$ and friction force $F_{\mathrm{e}}$ by electrostatic friction stimuli. The vibrotactile forces were calculated on the basis of nominal torque constant of the actuator and applied currents. The friction force was measured with DC voltages and a weight of $100 \mathrm{~g}(W \simeq 1 \mathrm{~N})$ placed on the aluminum pad pulled with a force gauge (DS2-20N, Imada, Japan, nominal resolution: $0.01 \mathrm{~N}$ ) at $1 \mathrm{~cm} / \mathrm{s}$. The values of $F_{\mathrm{v}}$ and $F_{\mathrm{e}}$ in Table 1 differ depending on the value of $\lambda$. A larger output force was needed for large values of $\lambda$ to perceive the texture stimulus. $F_{\mathrm{v}}$ was used to drive the panel as well; therefore, the actual vibrotactile force on the finger depends on the inertial resistance or acceleration of the panel. 
Table 1. Maximum Vibrotactile Force $F_{\mathrm{v}}$ and Electrostatic Friction Force $F_{\mathrm{e}}$ Measured with $W=1 \mathrm{~N}$

(a) $\lambda=0.5 \mathrm{~mm}$

\begin{tabular}{ccc}
\hline $\mathrm{Vb}: F r$ & $F_{\mathrm{v}}(\mathrm{N})$ & $F_{\mathrm{e}}(\mathrm{N})$ \\
\hline $10: 0$ & 2.1 & - \\
\hline $9: 1$ & 1.9 & 0.094 \\
\hline$\vdots$ & $\vdots$ & $\vdots$ \\
\hline $5: 5$ & 1.26 & 0.33 \\
\hline$\vdots$ & $\vdots$ & $\vdots$ \\
\hline $1: 9$ & 0.59 & 0.63 \\
\hline $0: 10$ & - & 0.70
\end{tabular}

(c) $\lambda=1.5 \mathrm{~mm}$

\begin{tabular}{ccc}
\hline $\mathrm{Vb}: \mathrm{Fr}$ & $F_{\mathrm{v}}(\mathrm{N})$ & $F_{\mathrm{e}}(\mathrm{N})$ \\
\hline $10: 0$ & 2.7 & - \\
\hline $9: 1$ & 2.5 & 0.35 \\
\hline$\vdots$ & $\vdots$ & $\vdots$ \\
\hline $5: 5$ & 1.8 & 0.63 \\
\hline$\vdots$ & $\vdots$ & $\vdots$ \\
\hline $1: 9$ & 1.1 & 0.91 \\
\hline $0: 10$ & - & 0.98
\end{tabular}

(e) $\lambda=2.5 \overline{\mathrm{mm}}$

\begin{tabular}{ccc}
\hline $\mathrm{Vb}: \mathrm{Fr}$ & $F_{\mathrm{V}}(\mathrm{N})$ & $F_{\mathrm{e}}(\mathrm{N})$ \\
\hline $10: 0$ & 4.2 & - \\
\hline $9: 1$ & 4.0 & 0.55 \\
\hline$\vdots$ & $\vdots$ & $\vdots$ \\
\hline $5: 5$ & 3.4 & 0.84 \\
\hline$\vdots$ & $\vdots$ & $\vdots$ \\
\hline $1: 9$ & 2.8 & 1.1 \\
\hline $0: 10$ & - & 1.19 \\
\hline
\end{tabular}

(b) $\lambda=1.0 \mathrm{~mm}$

\begin{tabular}{ccc}
\hline $\mathrm{Vb}: \mathrm{Fr}$ & $F_{\mathrm{v}}(\mathrm{N})$ & $F_{\mathrm{e}}(\mathrm{N})$ \\
\hline $10: 0$ & 2.5 & - \\
\hline $9: 1$ & 2.3 & 0.21 \\
\hline$\vdots$ & $\vdots$ & $\vdots$ \\
\hline $5: 5$ & 1.7 & 0.49 \\
\hline$\vdots$ & $\vdots$ & $\vdots$ \\
\hline $1: 9$ & 1.1 & 0.77 \\
\hline $0: 10$ & - & 0.84
\end{tabular}

(d) $\lambda=2.0 \mathrm{~mm}$

\begin{tabular}{ccc}
\hline $\mathrm{Vb}: F r$ & $F_{\mathrm{V}}(\mathrm{N})$ & $F_{\mathrm{e}}(\mathrm{N})$ \\
\hline $10: 0$ & 2.9 & - \\
\hline $9: 1$ & 2.74 & 0.38 \\
\hline$\vdots$ & $\vdots$ & $\vdots$ \\
\hline $5: 5$ & 2.1 & 0.7 \\
\hline$\vdots$ & $\vdots$ & $\vdots$ \\
\hline $1: 9$ & 1.46 & 1.02 \\
\hline $0: 10$ & - & 1.1
\end{tabular}

(f) $\lambda=3.0 \mathrm{~mm}$

\begin{tabular}{ccc}
\hline $\mathrm{Vb}: F r$ & $F_{\mathrm{V}}(\mathrm{N})$ & $F_{\mathrm{e}}(\mathrm{N})$ \\
\hline $10: 0$ & 5.0 & - \\
\hline $9: 1$ & 4.8 & 0.62 \\
\hline$\vdots$ & $\vdots$ & $\vdots$ \\
\hline $5: 5$ & 4.2 & 0.97 \\
\hline$\vdots$ & $\vdots$ & $\vdots$ \\
\hline $1: 9$ & 3.6 & 1.3 \\
\hline $0: 10$ & - & 1.4 \\
\hline
\end{tabular}

Part of the vibrotactile force is used to drive the top panel (weight: 283g) of the display. Vb. and Fr. indicate vibrotactile and frictional stimuli, respectively. The applied peak-to-peak voltages were $385,350,330,300,260$, and $200 \mathrm{~V}$ for $\lambda=3.0,2.5,2.0,1.5$, 1.0 , and $0.5 \mathrm{~mm}$, respectively, for the $0: 10$ combination.

\subsection{Participants}

Fifteen university students (mean age: 23,12 men, 3 women) participated in the test with written informed consent. They were not informed of the objective of the research.

\subsection{Procedure: An 11-alternative Forced-choice Task}

We investigated the composition ratio of the vibrotactile and frictional stimulation perceived to be realistic for each value of $\lambda$. The participants selected the most realistic stimulation among 11 types of stimuli for each value of $\lambda$ in comparison with the actual grating scale that they were allowed to experience freely. The 11 types of stimuli were randomly assigned to keys on a keyboard and could be switched by participants. From observations and interviews, their common strategies can be summarized as follows. First, participants experienced all stimuli 
and remembered a few stimuli that could be their answers. They were then carefully compared before a final judgment was made. They could experience virtual and actual stimuli as many times as desired. Typically, one trial required 2-3min, but the time for each trial was not limited.

We did not intend to strictly control the finger speed on the panel. The finger speed was calculated as the derivative of its position on the panel and shown on the personal computer for control. We specified the finger speed to be $10-20 \mathrm{~cm} / \mathrm{s}$, which was monitored by the experimenters during the task, to avoid extremely high or low speeds ${ }^{2}$. Each participant performed 18 trials (i.e., three trials for each of the six values of $\lambda$ ). The order of these trials was randomized. No visual images of the grating scales were presented on the texture display panel. Auditory cues were masked by wearing headphones playing pink noise.

\subsection{Results}

Figures 5(a)-5(f) show the ratios that the participants selected for the most realistic stimuli for every value of $\lambda$. Figure $5(\mathrm{~g})$ depicts the average over all values of $\lambda$.

We tested whether specific composition ratios were preferred over the others for each value of $\lambda$. All ratios should be equally perceived by the participants if no specific ratios were better than the others in terms of being more realistic. Hence, we conducted a goodness-of-fit test with the null hypothesis that all composition ratios are equally perceived by the participants (i.e., uniform probability distribution). The null hypothesis was not rejected when $\lambda$ was $0.5 \mathrm{~mm}$ but was rejected for $\lambda=1.0-3.0 \mathrm{~mm}$. Additional details are discussed as follows.

For $\lambda=0.5$, the most selected composition ratio was (vibrotactile:friction) $=(6: 4)$. Nonetheless, we did not find any statistically significant nonuniformity in the probability distribution $\left(\chi^{2}(10)=16.8, p>0.05\right)$.

For $\lambda=1.0$, the most selected composition ratio was (vibrotactile:friction) $=(8: 2)$ followed by $(0: 10)$ and $(10: 0)$. The responses were statistically nonuniform $\left(\chi^{2}(10)=19.3, p<0.05\right)$.

For $\lambda=1.5$, the most selected composition ratio was (vibrotactile:friction) $=(8: 2)$. After that, ratios $(10: 0)$ and (7:3) were equally selected. The responses were statistically nonuniform $\left(\chi^{2}(10)=25.2, p<0.01\right)$.

For $\lambda=2.0$, the most selected composition ratio was (vibrotactile:friction) $=(8: 2)$. The 11 composition ratios were significantly nonuniform $\left(\chi^{2}(10)=29.6, p<0.01\right)$.

For $\lambda=2.5$, the most selected composition ratio was (vibrotactile:friction) $=(8: 2)$ followed by (7:3). Stimulus conditions weighting vibrotactile stimuli were preferred to friction-weighted conditions. The 11 composition ratios were significantly nonuniform $\left(\chi^{2}(10)=24.7, p<0.01\right)$.

For $\lambda=3.0$, the most selected composition ratio was (vibrotactile:friction) $=(8: 2)$. Similar to $\lambda=2.5$, the vibrotactile-weighted stimuli were mainly selected by participants. The 11 composition ratios were not significantly uniform $\left(\chi^{2}(10)=24.2, p<0.01\right)$.

Figure $5(\mathrm{~g})$ shows the averaged selection of each composition ratio for all spatial wavelengths. Across all values of $\lambda$, the most selected composition ratio was (vibrotactile:friction) $=(8: 2)$, where the selection ratio of 0.22 was greater than the chance level $\left(z_{0}=7.5, p<0.001\right)$. The selection of composition ratios 10:0 and 0:10 followed that of $8: 2$, which might have been because these two types of stimuli were carefully tuned by experienced people, whereas the stimulus strengths of the other ratios were automatically determined. In total, the ratios (vibrotactile:friction) $=8: 2,10: 0$, and 0:10 were selected 59,39 , and 33 times by the participants, respectively. Post-hoc tests suggested that 8:2 was selected more than 10:0 $(z=2.02, p<0.05)$ and 0:10 $(z=2.62, p<0.01)^{3}$.

The results were divided depending on the spatial wavelengths of the grating scales. The participants were likely to select 8:2 or a similar ratio when $\lambda$ was large, whereas such trends were not strong when $\lambda=0.5 \mathrm{~mm}$. We discuss the possible explanations of these observed responses later.

\footnotetext{
${ }^{2}$ This condition was also employed when determining the values of $A$ and $B$ mentioned in Section 4.2.3.

${ }^{3}$ Ideally, we should conduct multiple pair-wise tests among the conditions. However, this overly lowers the value of $\alpha$ for each pair-wise test. Regarding this point, our $m$-alternative forced-choice design remains to be improved.
} 


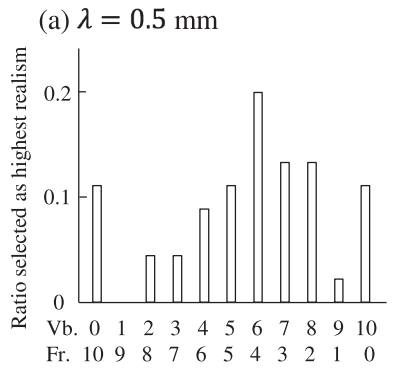

(b) $\lambda=1.0 \mathrm{~mm}$
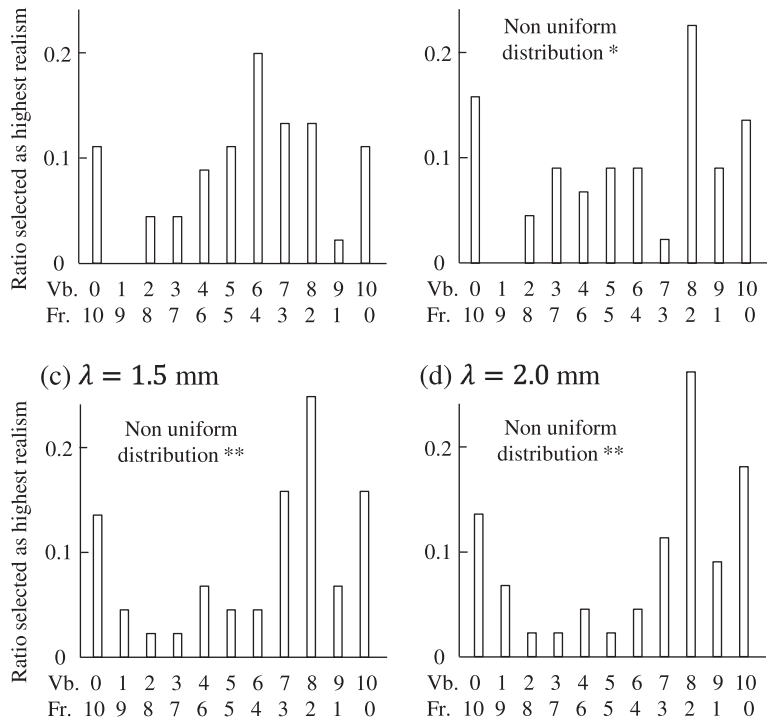

(e) $\lambda=2.5 \mathrm{~mm}$

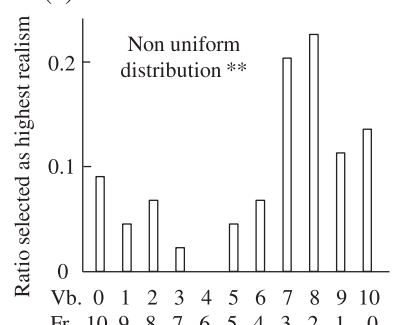

(d) $\lambda=2.0 \mathrm{~mm}$

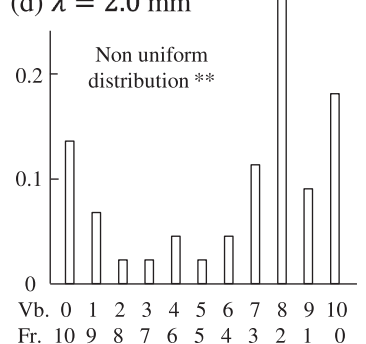

(f) $\lambda=3.0 \mathrm{~mm}$

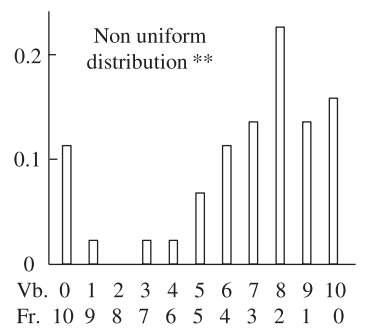

(g) Average

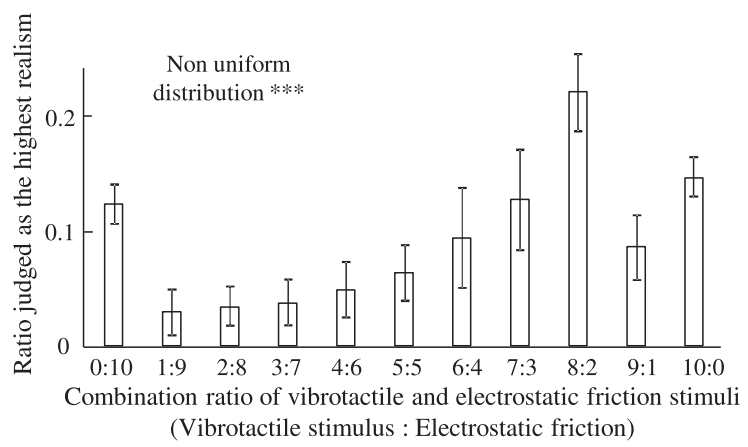

Fig. 5. Experimental results. The horizontal axes denote the composition ratios of the vibrotactile and electrostatic friction stimuli. The vertical axes denote the frequency selected as the most realistic stimulus. Fr. and Vb. represent friction and vibrotactile stimuli, respectively. The error bars in (g) are the $95 \%$ confidence intervals across panels (a)-(f). Asterisks * , **, and ${ }^{* * *}$ indicate statistically nonuniform ratios at $p<0.05,0.01$, and 0.001 , respectively. 


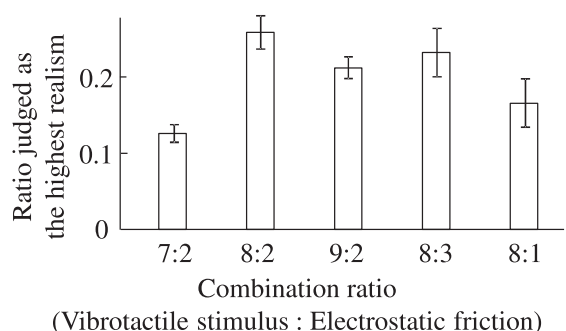

Fig. 6. Results of the follow-up experiment showing the average frequency of each stimulus condition selected as the most realistic stimulus. Error bars are the $95 \%$ confidence intervals.

\subsection{Follow-up Experiment}

In the above experiment, the composition ratio of $8: 2$ was significantly selected more than the vibrotactile- and electrostatic-only conditions, i.e., 10:0 and 0:10. However, one question is whether there exist conditions better than $8: 2$ when the sum of the two stimulus levels is not restricted to be 10. As a follow-up experiment, we compared five types of stimulus conditions near 8:2.

4.6.1 Stimuli and Tasks. Five stimulus conditions were prepared for each value of $\lambda=1.0,1.5,2.0,2.5$, and $3.0 \mathrm{~mm}$. The stimulus conditions were (vibrotactile:friction) $=(8: 2),(7: 2),(8: 1),(8: 3)$, and $(9: 2)$. Participants selected one of the five conditions in terms of realism. Each value of $\lambda$ was tested three times in total. Hence, 15 trials (three repetitions for each of the five values of $\lambda$ ) were conducted for each individual. Other conditions including sound masking, comparison with actual grating scales, and random stimulus assignment were the same as the task in Section 4.4. Ten university students who also participated in the experiments of Section 4.4 participated in this follow-up experiment.

4.6.2 Results. The selection ratios averaged across the five values of $\lambda$ are shown in Figure 6. We tested the uniformity of these average selection ratios, because no distinct trends were found across the five values of $\lambda$, and uniformity was not rejected $\left(\chi^{2}(4)=8.53, p>0.05\right)$. Even post-hoc pair-wise tests found a significant difference only between the combination ratios for 7:2 and 8:2 $\left(z_{0}=2.63, p<0.05\right)$. These results suggest that stimulus conditions similar to that of ( $8: 2)$ were equally selected, and they were not distinguished in terms of realism.

\section{DISCUSSION}

This experiment was intended to present the texture of a sinusoidal grating scale made of ABS resin. Note that the results may not be fully applied for other virtual textures or materials.

\subsection{Effectiveness of Either Vibrotactile or Electrostatic Friction Texture Display for Fine Roughness Texture}

For small spatial wavelengths $(\lambda=0.5 \mathrm{~mm})$, the virtual textures of the grating scales presented by each composition ratio were of the same quality in terms of their realism according to the uniformity test of frequencies. In other words, no specific composition ratio was considered to be more effective than the others.

These results may be explained by the perceptual mechanism of fine roughness textures. The exploration of actual and virtual fine textures causes high-frequency skin vibration. Whether a quality difference in perception exists between tangential and normal skin deformations at a high frequency is debatable, although they lead to different perceptual magnitudes or thresholds [8]. Assuming that the direction of the force that causes skin vibration is not significant for the perception of fine roughness and that the frequency and magnitude of skin vibration are rather important, all composition ratios were judged to be of equal quality. In other words, when 
the criterion was realism, the participants did not distinguish the vibratory texture presented by the normal vibrotactile and tangential friction forces.

Nonetheless, for $\lambda=0.5$, the ratio of $6: 4$ appeared to be more frequently selected than the other ratios, as shown in Figure 5(a). The composition ratios above the chance level were 4:6, 5:5, 6:4, and 8:2. At least, the combined stimulus did not deteriorate the presentation of fine roughness textures. In terms of even smaller values of $\lambda$, our setup may not be able to precisely deliver the stimulus because of the limited frequency responses of the display system.

\subsection{Effectiveness of Combined Vibrotactile and Electrostatic Stimuli for Roughness Scales with Large $\lambda$ Values}

The probability of selection for each stimulus was not uniform for grating scales with large spatial wavelengths $(\lambda \geq 1.0 \mathrm{~mm})$ in the first experiment. The ratio of $8: 2$ was mostly selected for every spatial wavelength. Stimuli with similar ratios such as 8:3 and 9:2 were equally selected in the follow-up experiment. In other words, the combination of vibrotactile and slight variable-friction stimuli is effective for presenting texture stimuli preferred in terms of realism, which is consistent with our earlier studies [22, 23], where combined stimuli were judged to be better for wavelengths of 1.0 and $2.0 \mathrm{~mm}$ than vibrotactile or electrostatic stimuli but not for wavelengths smaller than $1.0 \mathrm{~mm}$. The vibrotactile and electrostatic stimuli in [22, 23] correspond to 10:0 and 0:10 conditions in the present study, respectively.

For grating scales that are considered perceptually roughness-dominant, the vibrotactile stimuli that have been used for rendering roughness stimuli would be reasonable. However, the composition ratio of 8:2, which has a slight friction variation, would be selected with a high probability, because a variation in the friction force accrues when the finger pad slides on a real grating scale. This result implies that the deformation of the finger pad's skin along the normal direction mainly determined by surface asperities and the tangential deformation caused by friction may play different roles in texture perception $[18,40]$. One-dimensional quantities pertaining to skin deformation were measured in many studies related to the normal deformation $[6,56]$, tangential deformation [13, 45, 48, 57], or whole finger vibration [51], where skin deformation and texture perception were discussed. The information related to deformation or vibration in different dimensions is closely coupled, because they propagate in the fingertip. Hence, even unidimensional information may be satisfactory to be statistically linked with texture perception. However, the normal and tangential directions are different in terms of the mechanical characteristics comprising the mechanical impedance and vibration transfer properties [8, 59]. Normal and tangential skin surface stimuli have different influences on the mechanical receptors deep inside the skin, suggesting that combined normal and tangential deformations are different from either type of deformation in terms of texture perception.

\subsection{Further Aspects and Limitations of the Study}

Part of our research motivation, which was combining the effects of complementary texture displays, was clarified herein; however, some aspects remain to be studied. Although we used vibrotactile stimuli normal to the finger pad, it is intriguing to imagine the perceptual effect of the combination of tangential vibrotactile stimuli (e.g., References [21, 57]) and frictional stimuli. Another aspect is the generalization of the findings. Apparently, the preferred ratios differ among materials. In the case of finely polished ABS plastic used in this study, the ratio of 8:2 was selected; however, other ratios may be selected for sticky or slippery materials. We need a design guideline, because it is costly to repeat experiments similar to the one conducted in the present study for various types of materials. Note that inappropriate composition ratios deteriorate the realism of textures, because most combined stimuli were less preferred than vibrotactile- and electrostatic-only stimuli in our experiments.

Although we introduced a conductive pad to control the frictional conditions, this pad is unlikely to be used for commercial applications. Hence, for the practical applications with bare fingers, for example, feedback control of 
the frictional force is demanded to ensure that users are subjected to set stimulus forces. Further, the conditions of slippage may differ between when a fingertip slides on a flat panel and when a conductive pad slides on a flat panel, which may influence the realism of frictional textures.

\section{CONCLUSION}

We presented virtual roughness textures with a spatial wavelength ranging from 0.5 to $3.0 \mathrm{~mm}$. A tactile texture display that could present vibrotactile and electrostatic friction stimuli was used. In the experiment, the participants selected the stimulus that they perceived to be most realistic among the 11 types of combined vibrotactile and electrostatic stimuli. The two extreme stimuli among them were the vibrotactile- and friction-only stimuli, whereas the other nine stimuli were the combination of the two types of stimuli. The result was different depending on the spatial wavelength. We did not find a significant difference in the response for 11 types of stimuli for a relatively small wavelength $(\lambda=0.5 \mathrm{~mm})$. In the range of relatively large spatial wavelengths $(\lambda \geq 1.0 \mathrm{~mm})$, vibrotactile stimuli with slight electrostatic friction stimuli were effective for presenting virtual grating scales. These findings will contribute to the determination of the specifications of high-quality texture displays.

\section{REFERENCES}

[1] T. Aktar, J. Chen, R. Ettelaie, M. Holmes, and B. Henson. 2017. Human roughness perception and possible factors effecting roughness sensation. F. Text. Studies 48, 3 (2017), 181-192.

[2] Shuhei Asano, Shogo Okamoto, and Yoji Yamada. 2015. Vibrotactile stimulation to increase and decrease texture roughness. IEEE Trans. Hum.-Mach. Syst. 45, 3 (2015), 393-398.

[3] S. Ballesteros, J. M. Reales, L. Ponce de León, and B. García. 2005. The perception of ecological textures by touch: Does the perceptual space change under bimodal visual and haptic exploration? Proceedings of IEEE World Haptics Conference. 635-638.

[4] Olivier Bau, Ivan Poupyrev, Ali Israr, and Chris Harrison. 2010. TeslaTouch: Electrovibration for touch surfaces. In Proceedings of 23rd Annual ACM Symposium on User Interface Software and Technology. ACM, 283-292.

[5] S. J. Bensmaïa. 2009. Texture from touch. Scholarpedia 4, 8 (2009), 7956.

[6] S. J. Bensmaïa and M. Hollins. 2005. Pacinian representations of fine surface texture. Percept. Psychophys. 67, 5 (2005), $842-854$.

[7] W. M. Bergmann Tiest. 2010. Tactual perception of material properties. Vision Res. 50, 24 (2010), 2775-2782.

[8] James Biggs and Mandayam A. Srinivasan. 2002. Tangential versus normal displacements of skin: Relative effectiveness for producing tactile sensations. Proceedings of the IEEE Hpatics Symposium, 121-128.

[9] D. G. Caldwell and C. Gosney. 1993. Multi-modal tactile sensing and feedback (Tele-Taction) for enhanced tele-manipulator control. Proceedings of IEEE/RSF International Conference on Intelligent Robots and Systems (1993), 1487-1494.

[10] F. R. Clarke. 1957. Constant ratio rule for confusion matrices in speech communication. J. Acoust. Soc. Amer. 29, 6 (1957), $751-720$.

[11] Heather Culbertson and Katherine J. Kuchenbecker. 2017. Importance of matching physical friction, hardness, and texture in creating realistic haptic virtual surfaces. IEEE Trans. Haptics1 (2017), 63-74.

[12] Kenneth Duvefelt, Ulf Olofsson, Carl Michael Johannesson, and LisaSkedung. 2016. Model for contact between finger and sinusoidal plane to evaluate adhesion and deformation component of friction. Tribol. Int. 96 (2016), 389-394.

[13] Ramona Fagiani, Francesco Massi, Eric Chatelet, Yves Berthier, and Adnan Akay. 2011. Tactile perception by friction induced vibrations. Tribol. Int. 44 (2011), 1100-1110.

[14] Yohei Fujii, Shogo Okamoto, and Yoji Yamada. 2016. Friction model of fingertip sliding over wavy surface for friction-variable tactile feedback panel. Adv. Robot. 30 (2016), 1341-1353.

[15] Sofiane Ghenna, Eric Vezzoli, Christophe Giraud-Audine, Frederic Giraud, Michel Amberg, and Betty Lemaire-Semail. 2017. Enhancing variable friction tactile display using an ultrasonic travelling wave. IEEE Trans. Haptics 10, 2 (2017), 296-301.

[16] S. Guest, J. M. Dessirier, A. Mehrabyan, F. McGlone, G. Essick, G. Gescheider, A. Fontana, R. Xiong, R. Ackerley, and K. Blot. 2011. The development and validation of sensory and emotional scales of touch perception. Atten. Percept. Psychophys. 73 (2011), 531-550.

[17] Taku Hachisu and Hiroyuki Kajimoto. 2017. Vibration feedback latency affects material perception during rod tapping interactions. IEEE Trans. Haptics 10, 2 (2017), 288-295.

[18] Hikaru Hasegawa, Shogo Okamoto, Elfekey Hatem, and Yoji Yamada. 2018. Stochastic relationships between the normal and shear interaction forces during tactile exploration of textures. In Proceedings of IEEE International Conference on Systems, Man, and Cybernetics.

[19] M. Hollins, S. J. Bensmaïa, K. Karlof, and F. Young. 2000. Individual differences in perceptual space for tactile textures: Evidence from multidimensional scaling. Atten. Percept. Psychophys. 62, 8 (2000), 1534-1544.

[20] M. Hollins and S. R. Rinser. 2000. Evidence for the duplex theory of tactile texture perception. Atten. Percept. Psychophys. 62, 4 (2000), 695-705. 
[21] Akihiro Imaizumi, Shogo Okamoto, and Yoji Yamada. 2017. Friction perception resulting from laterally vibrotactile stimuli. Robomech. 7. 4 (2017), 12.

[22] Ken Ito, Shogo Okamoto, Hatem Elfekey, Hiroyuki Kajimoto, and Yoji Yamada. 2017. A texture display using vibrotactile and electrostatic friction stimuli surpasses one based on either type of stimulus. In Proceedings of IEEE International Conference on Systems, Man, and Cybernetics. 2343-2348.

[23] Ken Ito, Shogo Okamoto, Hatem Elfekey, and Yoji Yamada. 2018. High-quality texture display: The use of vibrotactile and variablefriction stimuli in conjunction. In Haptic Interaction, Shoichi Hasegawa, Masashi Konyo, Ki-Uk Kyung, Takuya Nojima, and Hiroyuki Kajimoto (Eds.). Springer, 125-130.

[24] M. Janko, M. Wiertlewski, and Y. Visell. 2018. Contact geometry and mechanics predict friction forces during tactile surface exploration. Nature Sci. Rep. 8 (2018), 4868.

[25] P. Kammermeier, A. Kron, J. Hoogen, and G. Schmidt. 2004. Display of holistic haptic sensations by combined tactile and kinesthetic feedback. Presence 13, 1 (Feb. 2004), 1-15. DOI : https://doi.org/10.1162/105474604774048199

[26] Seung-Chan Kim, Ali Israr, and Ivan Poupyrev. 2013. Tactile rendering of 3D features on touch surfaces. Proceedings of ACM Symposium on User Interface Software and Technology (2013), 531-538.

[27] Seung Chan Kim, Ali Israr, and Ivan Poupyrev. 2013. Tactile rendering of 3D features on touch surfaces. In Proceedings of 26th Annual ACM Symposium on User Interface Software and Technology. 531-538.

[28] Roberta L. Klatzky, Dianne Pawluk, and Angelika Peer. 2013. Haptic perception of material properties and implications for applications. Proc. IEEE 101, 9 (2013), 2081-2092.

[29] M. Konyo, S. Tadokoro, A. Yoshida, and N. Saiwaki. 2005. A tactile synthesis method using multiple frequency vibrations for representing virtual touch. In Proceedings of IEEE/RSf International Conference on Intelligent Robots and Systems. 3965-3971.

[30] Tadatoshi Kurogi, Masano Nakayama, Katsunari Sato, Sho Kamuro, Charith Lasantha Fernando, Masahiro Furukawa, Kouta Minamizawa, and Susumu Tachi. 2013. Haptic transmission system to recognize differences in surface textures of objects for telexistence. In Proceedings of IEEE Virtual Reality. 137-138.

[31] Vincent Levesque, Ali Modarres, Juan Manuel Cruz-Hernandez, Amaya Becvar Weddle, David M. Birnbaum, and Danny A. Grant. 2017. Systems and methods for generating friction and vibrotactile effects, U.S. Patent US9639158B2.

[32] Vincent Lévesque, Louise Oram, Karon MacLean, Andy Cockburn, Nicholas D. Marchuk, Dan Johnson, J. Edward Colgate, and Michael A. Peshkin. 2011. Enhancing physicality in touch interaction with programmable friction. In Proceedings of ACM SIGCHI Conference on Human Factors in Computing Systems. 2481-2490.

[33] R. D. Luce. 2005. Individual Choice Behavior: A Theoretical Analysis. Dover Publications.

[34] Joseph Mullenbach, Michael Peshkin, and J. Edward Colgate. 2017. eShiver: Lateral Force feedback on fingertips through oscillatory motion of an electroadhesive surface. IEEE Trans. Haptics 10, 3 (2017), 358-370.

[35] T. Nakamura and A. Yamamoto. 2016. A multi-user surface visuo-haptic display using electrostatic friction modulation and capacitivetype position sensing. IEEE Trans. Haptics 9, 3 (2016), 311-322.

[36] S. Okamoto. 2014. VR-MDS: Multidimensional scaling for classification tasks of virtual and real stimuli. Atten. Percept. Psychophys. 76, 3 (2014), 877-893.

[37] Shogo Okamoto, Hikaru Nagano, and Yoji Yamada. 2013. Psychophysical dimensions of tactile perception of textures. IEEE Transactions on Haptics 6, 1 (2013), 81-93.

[38] A. M. Okamura, J. T. Dennerlein, and R. D. Howe. 1998. Vibration feedback models for virtual environments. In Proceedings of IEEE International Conference on Robotics and Automation, Vol. 1. 674-679.

[39] R. H. Osgouei, J. R. Kim, and S. Choi. 2017. Improving 3D Shape recognition withelectrostatic friction display. IEEE Trans. Haptics 10, 4 (2017), 533-544.

[40] Jonathan Platkiewicz, Alessandro Mansutti, Monica Bordegoni, and Vincent Hayward. 2014. Recording device for natural haptic textures felt with the bare fingertip. In Haptics: Neuroscience, Devices, Modeling, and Applications (Lecture Notes in Computer Science), Malika Auvray and Christian Duriez (Eds.), Vol. 8618. Springer, Berlin, 521-528.

[41] Dongbum Pyo, Semin Ryu, Seung-Chan Kim, and Dong-Soo Kwon. 2014. A new surface display for 3D haptic rendering. In Haptics: Neuroscience, Devices, Modeling, and Applications, Malika Auvray and Christian Duriez (Eds.). Springer Berlin, 487-495.

[42] Gabriel Robles-De-La-Torre and Vincent Hayward. 2001. Force can overcome object geometry in the perception of shap through active touch. Nature 412 (2001), 445-448.

[43] Semin Ryu, Dongbum Pyo, Byung Kil Han, and Dong Soo Kwon. 2018. Simultaneous representation of texture and geometry on a flat touch surface. In Haptic Interaction, Shoichi Hasegawa, Masashi Konyo, Ki-Uk Kyung, Takuya Nojima, and Hiroyuki Kajimoto (Eds.). Springer, 83-86.

[44] Semin Ryu, Dongbum Pyo, Soo-Chul Lim, and Dong-Soo Kwon. 2018. Mechanical vibration influences the perception of electrovibration. Sci. Rep. 8 (2018), 4555.

[45] Shunsuke Sato, Shogo Okamoto, Yoichiro Matsuura, and Yoji Yamada. 2017. Wearable finger pad deformation sensor for tactile textures in frequency domain by using accelerometer on finger side. Robomech. f. 4 (2017), 19. 
[46] H. Shirado and T. Maeno. 2005. Modeling of human texture perception for tactile displays and sensors. Proceedings of IEEE World Haptics Conference (2005), 629-630.

[47] L. Skedung, K. Danerlöv, U. Olofsson, C. M. Johannesson, M. Aikala, J. Kettle, M. Arvidsson, B. Berglund, and M. W. Rutland. 2011. Tactile perception: Finger friction, surface roughness and perceived coarseness. Tribol. Int. 44 (2011), 505-512.

[48] A. M. Smith, C. E. Chapman, M. Deslandes, J. S. Langlais, and M. P. Thibodeau. 2002. Role of friction and tangential force variation in the subjective scaling of tactile roughness. Exp. Brain Res. 144, 2 (2002), 211-223.

[49] T. A. Smith and J. L. Gorlewicz. 2017. Hue: A hybrid ultrasonic and electrostatic variable friction touchscreen. In Proceedings of IEEE World Haptics Conference. 635-640.

[50] A. Sutu, E. Meftah, and E. Chapman. 2013. Physical determinants of the shape of the psychophysical curve relating tactile roughness to raised-dot spacing: Implications for neural coding of roughness. f. Neurophysiol. 109, 5 (2013), 1403-1415.

[51] Yoshihiro Tanaka, Wouter M. Bergmann Tiest, Astrid M. L. Kappers, and Akihito Sano. 2014. Contact force and scanning velocity during active roughness perception. PLOS One 9, 3 (2014), e93363. DOI : https://doi.org/10.1371/journal.pone.0093363

[52] M. M. Taylor and S. J. Lederman. 1975. Tactile roughness of grooved surfaces: A model and the effect of friction. Atten. Percept. Psychophys. 17, 1 (1975), 23-36.

[53] J. T. Townsend and D. E. Landon. 1982. An experimental and theoretical investigation of the constant-ratio rule and other models of visual letter confusion. f. Math. Psychol. 25 (1982), 119-162.

[54] Julien van Kuilenburg, Marc A. Masen, and Emile van der Heide. 2015. A review of fingerpad contact mechanics and friction and how this affects tactile perception. Proc. Inst. Mech. Eng. Part f: f. Eng. Tribol. 229, 3 (2015), 243-258.

[55] Yasemin Vardar, Aykut Isleyen, Muhammad K. Saleem, and Cagatay Basdogan. 2017. Roughness perception of virtual textures displayed by electrovibration on touch screens. In Proceedings of the IEEE World Haptics Conference. 263-268. DOI : https://doi.org/10.1109/WHC. 2017.7989912

[56] Alison I. Webera, Hannes P. Saala, Justin D. Lieberb, Ju-Wen Chenga, Louise R. Manfredia, John F. Dammann III, and Sliman J. Bensmaïa. 2013. Spatial and temporal codes mediate the tactile perception of natural textures. Proc. Natl. Acad. Sci. U.S.A. 110, 42 (2013), 1710717112.

[57] Michael Wiertlewski, José Lozada, and Vincent Hayward. 2011. The spatial spectrum of tangential skin displacement can encode tactual texture. IEEE Trans. Robot. 27, 3 (2011), 461-472.

[58] L. Winfield, J. Glassmire, J. E. Colgate, and M. Peshkin. 2007. T-PaD: Tactile pattern display through variable friction reduction. Proceedings of the 2nd foint EuroHaptics Conference and Symposium on Haptic Interfaces for Virtual Environment and Teleoperator Systems (2007), 421-426.

[59] John Z. Wu, Daniel E. Welcome, Kristine Krajnak, and Ren G. Dong. 2007. Finite element analysis of the penetrations of shear and normal vibrations into the soft tissues in a fingertip. Med. Eng. Phys. 29 (2007), 718-727.

[60] T. Yamauchi, S. Okamoto, M. Konyo, Y. Hidaka, T. Maeno, and S. Tadokoro. 2010. Real-time remote transmission of multiple tactile properties through master-slave robot system. In Proceedings of IEEE International Conference on Robotics and Automation. 1753-1760. DOI : https://doi.org/10.1109/ROBOT.2010.5509926

[61] T. Yoshioka, S. J. Bensmaïa, J. C. Craig, and S. S. Hsiao. 2007. Texture perception through direct and indirect touch: An analysis of perceptual space for tactile textures in two modes of exploration. Somatosens. Motor Res. 24, 1-2 (2007), 53-70.

Received January 2019; revised May 2019; accepted May 2019 